\title{
Bone Marrow Cells Obtained from Old Animals Differ from the Young Animals Cells in Their Ability to Divide and in Response to the Presence of Liver Fibrosis in Primary Culture
}

\author{
S. L. Ohiienko1, A. I. Bozhkov1*, A. Yu. Bondar'1, E. G. Ivanov'1, I. A. Ionov² \\ ${ }^{1}$ Research Institute of Biology, V.N. Karazin Kharkiv National University, Kharkov, Ukraine \\ ${ }^{2}$ G. S. Skovoroda Kharkiv National Pedagogical University, Kharkov, Ukraine \\ Email: *bozhkov@univer.kharkov.ua
}

How to cite this paper: Ohiienko, S.L., Bozhkov, A.I., Bondar, A.Yu., Ivanov, E.G. and Ionov, I.A. (2019) Bone Marrow Cells Obtained from Old Animals Differ from the Young Animals Cells in Their Ability to Divide and in Response to the Presence of Liver Fibrosis in Primary Culture. Advances in Aging Research, 8, 14-27.

https://doi.org/10.4236/aar.2019.81002

Received: December 27, 2018

Accepted: January 28, 2019

Published: January 31, 2019

Copyright $\odot 2019$ by author(s) and Scientific Research Publishing Inc. This work is licensed under the Creative Commons Attribution International License (CC BY 4.0).

http://creativecommons.org/licenses/by/4.0/

\begin{abstract}
Dynamics of bone marrow cells number (BMC) in the primary culture isolated from young ( 3 months) and old (20 months) Wistar rats was investigated. Proliferative activity of BMC of old animals was 2 times higher than that of young animals in the primary culture. Such superiority of the proliferative activity of BMC in the primary culture obtained from old animals is associated with the ability to actively divide lymphocytes and longer "lifespan" of segmented neutrophils obtained from old animals. It should be noted, that the lymphocytes of young animals did not proliferate in the primary culture. The content of intracellular calcium in BMC in the cells of old animals was 3 times higher compared with cells of young animals, which revealed the relationship of intracellular calcium and proliferative activity of BMC. Induction of liver fibrosis led to an increase in the lymphocyte content in young animals by $167 \%$, and in old ones only by $26 \%$, while the lymphocytes of young animals acquired the ability to proliferate in the primary culture. It has been suggested that such differences in the behavior of BMC in primary culture obtained in young and old animals reflect differences in the BMC microenvironment of young and old animals, which leads to changes in the epigenetic-metabolic characteristics of BMC.
\end{abstract}

\section{Keywords}

Bone Marrow, Aging, Proliferation, Lymphocyte, Intracellular $\mathrm{Ca}^{2+}$

\section{Introduction}

One of the significant achievements of the biology of the XX century can be con- 
sidered the selection, research and development of methods for the cultivation of stem cells [1] [2]. These studies led to the formation of regenerative medicine, the prospects for which have high hopes [3] [4].

Moreover, stem cells are interesting for gerontologists. As a result, new hypotheses have emerged. According to that, stem cells can become the very means that can accelerate the regeneration of organs, and normalize the ability of an adult organism to adapt to various extreme influences [5] [6].

Indeed, stem cells can differentiate into any type of cells depending on the characteristics of the microenvironment [7] [8]. Currently, a sufficiently large amount of data was obtained. They showed a therapeutic effect after transfusion of stem cells [9] [10]. The model of Cu-induced liver fibrosis showed a pronounced therapeutic effect after the administration of human embryonic stem cells to rats [11]. These studies suggest a decrease or "exhaustion" of the stem cell pool during ontogenesis. At the same time, the introduction of an additional number of stem cells provides a compensatory effect. Indeed, there are quite a large number of works in which it has been shown that the number of hematopoietic stem cells in the bone marrow decreases in the aging organism and more often than in young animals [12] [13] and other pathologies associated with the functions of immunocompetent cells and blood cells.

Along with this, there are data indicating that the number of stem cells in bone marrow does not change with age and their proliferative potential is not reduced [14] [15].

Currently, the study of the quantitative and qualitative characteristics of the bone marrow cells (BMC) in ontogenesis is an important gerontological task. In addition, further development strategies of gerontological and geriatric studies depend on the knowledge of the answer to this question.

In this regard, the study of BMC is of particular importance. They provide blood formation, synthesis of immunocompetent cells and are the source of mesenchymal stem cells. In the study of a pool of stem cells, the method of determining stem cells in the bone marrow or other depots by the presence of appropriate cellular markers [16], or after long-term cultivation of previously isolated stem cells [17] is most often used. At the same time, the interaction of different cell types in the bone marrow is not taken into account, i.e. age characteristics of cellular niches and possible epigenetic changes that may occur during ontogenesis are not taken into account. Without knowledge of these features of BMC, it is impossible to assess the role of stem cells in ontogenesis and influence the behavior of BMC.

We believe that different experimental models should be used to solve these complex issues. This is of great interest in understanding the regulatory systems of cells.

In this regard, primary culture of BMC may be successful model. The following is characteristic of the primary culture of BMC: a good analogy of cells with an in vivo system, since the culture maintains a completely heterogeneous pop- 
ulation of BMC, which provides possible intercellular interactions. During primary cultivation, the epigenetic pattern formed in the in vivo system is still preserved; in a primary culture, it is possible to evaluate the potential "lifespan" of cells in a culture and determine the factors that influence it, and the possible relationship of the proliferative activity of BMC with calcium content.

In this regard, the ability of BMC to proliferate, which were isolated from young ( 3 months) and old (20 months) animals in the primary culture (from 1 to 4 days of growth), as well as "lifespan" (preservation time in culture) of lymphocytes and neutrophils obtained from the BMC of animals were determined.

\section{Materials and Methods}

\subsection{Experimental Scheme of Preparation of Animals for Research}

A total of 32 mature male Wistar rats were separated into experiments. These rats of two different age groups ( 3 and 20 month old) were obtained from Research Institute of Biology, V.N. Karazin Kharkov National University (Kharkov, Ukraine). They were housed in a temperature controlled room $\left(20^{\circ} \mathrm{C}-24^{\circ} \mathrm{C}\right)$ and adapted to a $12 \mathrm{~h}$ light/12 $\mathrm{h}$ dark cycle. The animals were given free access to food and water before and during the study. All experimental procedures employed were approved by and conducted in accordance with bioethical rules [18] and with due consideration to circadian rhythms for the formation of biological responses. The experiment protocol for animal should be in accordance with the standard animal welfare guideline, and the permission should be obtained from Ethics Committee. For 24 hours preceding isolation of BMC, animals did not receive any food. Removing animals from the experiment was always carried out from 8 to 10 a.m. local time.

The rats were divided into two age groups: 16 young (3-month-old, body weight $150-200 \mathrm{~g}$ ) and 16 old (20-month-old, body weight $350-400 \mathrm{~g})$ ones. In each age group, animals were divided into groups: an intact control group ( $\mathrm{n}=$ 8 ) and a group with liver fibrosis $(\mathrm{n}=8)$. Rat models of $\mathrm{Cu}$-induced liver fibrosis were established by triple intraperitoneally injection of copper sulphate in doses $1 \mathrm{mg} / 100 \mathrm{~g}$ of liver weight with 48 hours intervals between injections, as in investigation [19]. Rats injected with saline $(0.95 \% \mathrm{NaCl})$ served as a control group.

\subsection{Isolation and Culture of Bone Marrow Cells in Vitro}

BMC were isolated from the 2 femoral bones of the rat by the method [20] and cultured in medium DMEM (Biowest, USA) with antibiotics (1\% gentamicin and $1 \%$ streptomycin) and $20 \%$ inactivated fetal calf serum (Gibco, USA) in plastic cups ( $35 \mathrm{~mm}$, Nunk, Denmark). Culture under standard conditions was carried out at $37^{\circ} \mathrm{C}$ in the $5 \%$ atmosphere. The number of cells and their morphotypes were determined daily from 1 to 4 days of cultivation. The culture medium was left unchanged. The initial concentration of BMC during culture always was 2 million cells $/ \mathrm{ml}$. 


\subsection{Determination of Lymphocytes and Neutrophils Number}

The count of BMC in animals was carried out as described in the paper [21]. Determination of lymphocytes and neutrophils was carried out immediately after a suspension of BMC was obtained, as well as on the 2 nd and 4 th days of culturing as described in the paper [22]. Cytological preparations were stained by Romanovsky-Giemsa, analyzed at 100-fold magnification with the Zeiss Primo Stari LED microscope (Germany).

\subsection{Determination of Calcium Ions in Bone Marrow Cells}

Intracellular calcium content was performed using a specific Fluo-3 probe. 10 $\mu \mathrm{g} / \mathrm{ml}$ of Fluo-3 prepared on $3.03 \mathrm{mM}$ phosphate buffer, containing $2.89 \mathrm{mM}$ $\mathrm{CaCl}_{2}$, was added to a suspension containing 2 million cells per ml. The cell suspension with the dye was incubated for 15 minutes at $24^{\circ} \mathrm{C}$, as described [23]. Cytological preparations were analyzed at 100-fold magnification with the confocal laser scanning microscopy of LSM 510 META Carl Zeiss (Germany).

\subsection{Statistical Analysis}

Average deviation, standard deviation, standard error of the mean, and sample size were used as characteristics of the samples. The statistical significance of the differences between the two groups of data was assessed using the nonparametric Mann-Whitney U-test. The statistical processing of the results was carried out using the STATISTICA 10 software. Differences between the control and trial data were considered reliable at $\mathrm{p} \leq 0.05$.

\section{Results}

Suspension of BMC obtained in young and old animals was cultured on DMEM medium with the addition of $20 \%$ calf serum so that $2 \times 10^{6}$ cells per ml were contained. It was found that if BMC were obtained from young animals, then after 24 hours of incubation, their number increased by $60 \%$ (Figure 1 ).

If BMC were obtained from old animals, over the same time, under the same conditions, their number increased by $112 \%$, i.e. almost 2 times more compared with young animals (Figure 1). The amount of BMC in the culture increased to 3 days of growth, after which the culture reached a stationary level, and then the cells began to die, which corresponds to the standard behavior of cells in primary cultures [24]. After 24 hours of cultivation, the growth rate of the culture obtained from old animals was slowed down faster than the cell culture of young animals. However, differences remained for BMC obtained in older animals compared with young animals (Figure 1).

Consequently, the culture of BMC, obtained in old animals, exceeded, in terms of growth, the cell culture of young animals.

The BMC population is represented by different cell types: mesenchymal stem cells, committed and differentiated cells (lymphocytes, neutrophils, myelocytes, monocytes, eosinophils, basophils), as well as a large number of cells at different 


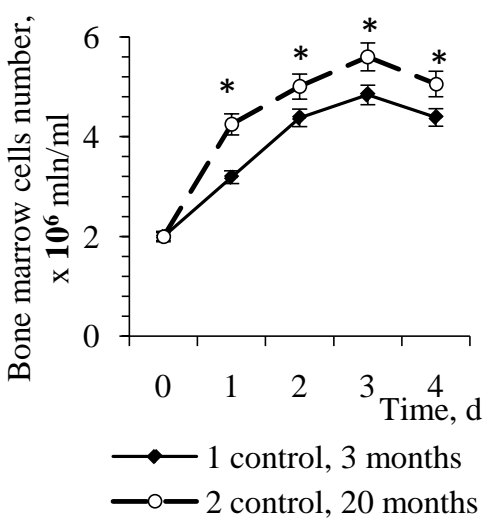

Figure 1. The number of BMC obtained from 1 to 4 days of in vitro culture from intact 3 -month old $(\longrightarrow-)$ and intact 20 month old $(-\infty)$ ) animals. Data percentage is expressed as mean, obtained of three independent experiments \pm S.E.M, $n=8$ per group; $\mathrm{p}$ $\leq 0.05$ between young and old animals are determined by ${ }^{*}$, by Mann-Whitney's U-test.

stages of differentiation. Stem cells have the highest proliferative activity, although lymphocytes also retain the ability to divide in culture [25]. However, the intensity of lymphocyte division in a primary culture depends on a large number of endogenous and exogenous factors.

It turned out that if BMCs were obtained from young animals and transferred to culture, the number of lymphocytes after 2 days of cultivation decreased by $24 \%$ from the initial amount. After 4 days of cultivation, their amount decreased by $50 \%$ from the original, i.e. there was a linear decrease in the number of lymphocytes, if they were isolated from young animals (Figure 2(A)).

In that case, if lymphocytes were obtained from old animals, their number, on the contrary, increased linearly up to 4 days of cultivation. It should be noted that the number of lymphocytes on the 4 th day was increased by $48 \%$, compared with the initial number (Figure 2(A)).

It should be noted that in the population of BMC obtained from old animals, there were 2 times more lymphocytes compared with young animals (Figure 2(a)).

As it is known, $15 \%-24 \%$ of stab neutrophils are present in the bone marrow. It is known that with the "release" of band neutrophils into the blood, they have a short "lifespan" from 5 to 48 hours. The transfer of BMC to the primary culture resulted in a linear decrease in the number of band neutrophils from the beginning of cultivation to 4 days, if BMC were obtained from young animals (Figure 2(B)). In that case, if BMCs were obtained from old animals, their number decreased at a slower rate compared with young animals (Figure 2(B)).

Consequently, the "lifespan" of stab neutrophils obtained from old animals was longer in culture compared with those obtained from young animals.

The total number of stab neutrophils in the bone marrow of old animals was $6.7 \%$ more than in young animals (Figure $2(\mathrm{~b})$ ). In the process of differentiation, stab neutrophils turn into mature segmented neutrophils, which are capable of active phagocytosis [26]. 


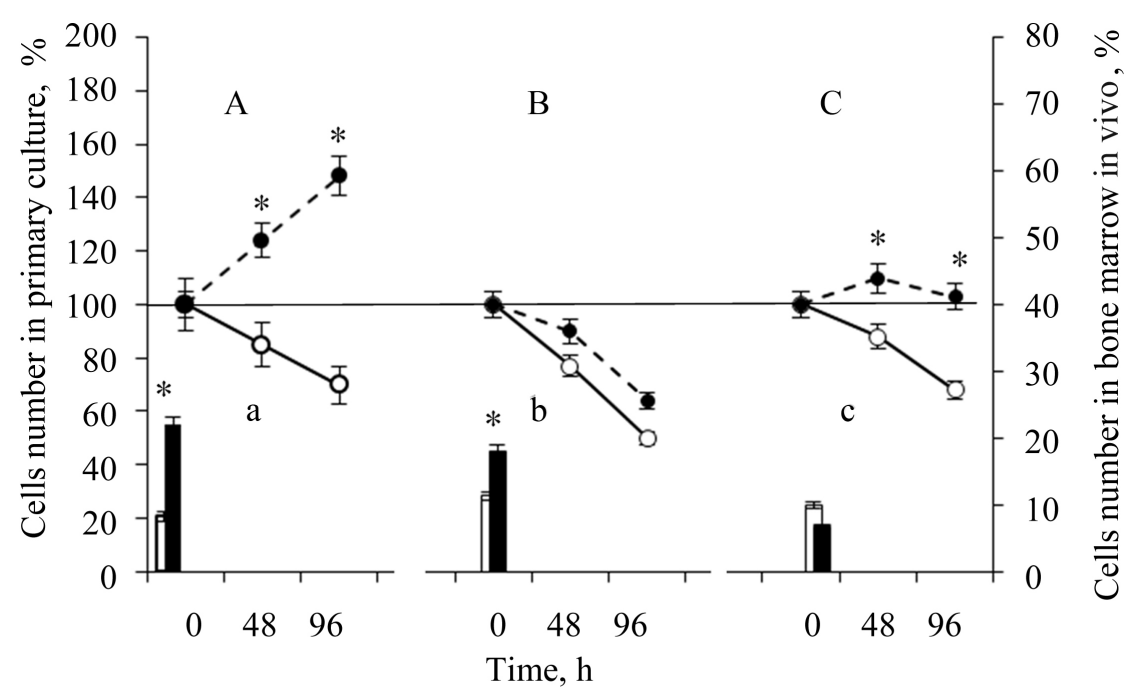

- - - 3 months $\longrightarrow-20$ months

Figure 2. The number of lymphocytes (A), band neutrophils (B) and segmented neutrophils (C) in the primary culture of BMC at 48 and 96 hours of cultivation obtained from intact young ( 3 months) and old (20 months) animals, and the number of lymphocytes (a), band neutrophils (b) and segmented neutrophils (c) contained in the bone marrow in vivo of young ( ${ }^{2}$ ) and old ( $)$ ) animals. ${ }^{\star}$ Data percentage is expressed as mean, obtained of three independent experiments \pm S.E.M, $\mathrm{n}=8$ per group; variants with $\mathrm{p} \leq$ 0.05 between young and old animals are determined by *, by Mann-Whitney's U-test.

It was found that the number of segmented neutrophils of young animals in the primary culture of BMC decreased and on the 4th day of cultivation, their number was $32 \%$ less (Figure 2(C)). In the event that BMCs were obtained from old animals, the number of segmented neutrophils in the culture remained almost unchanged during the 4 days of cultivation (Figure 2(C)). It should be noted that the total number of segmented neutrophils in the bone marrow of old animals was reduced by $3.3 \%$ compared with the bone marrow of young animals (Figure 2(c)). Consequently, a large proliferative activity of BMC in the primary culture of old animals is associated with a high proliferative activity of lymphocytes in old animals and a lower death rate of stab and segmented neutrophils in the primary culture.

Such different "behavior" of BMC obtained from old and young animals under the same cultivation conditions (the same medium, the same number of cells in culture and the same cultivation conditions) may indicate differences in the metabolic and epigenetic characteristics of BMC animals of different ages.

In the next series of experiments, the content of calcium ions in the BMC of young and old animals was determined. At the same time, we proceeded from the fact that an increase in the intracellular calcium content leads to the activation of cell proliferation [27]. It was found that the content of calcium ions, judging by the activity of Fluo-3 luminescence in BMC obtained from old animals was 3 times higher than in BMC from young animals (Figure 3 ).

It is obvious that an increase in intracellular calcium content, on the one hand, 
A

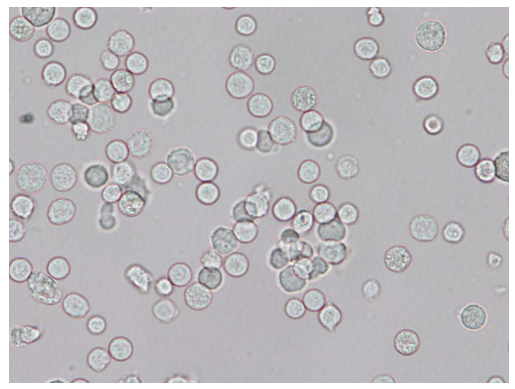

C

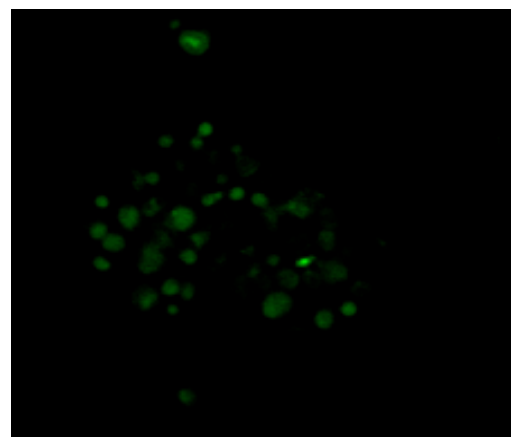

$\mathrm{E}$

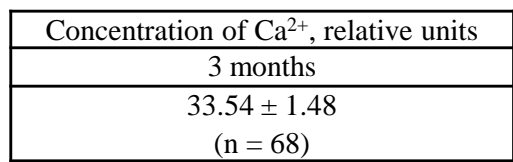

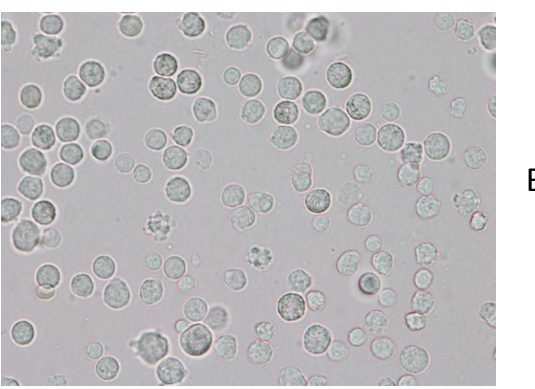

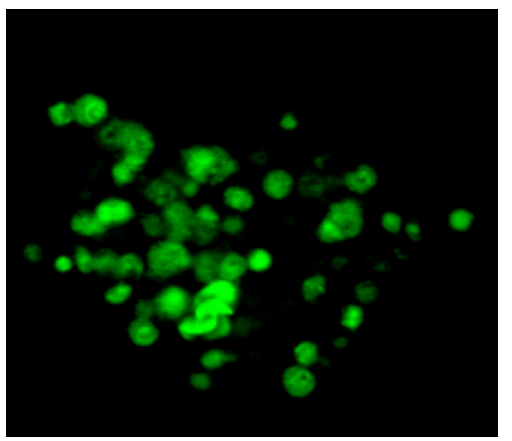

Concentration of $\mathrm{Ca}^{2+}$, relative units

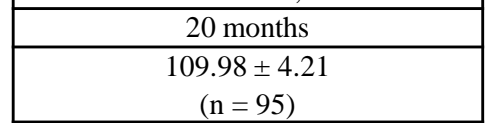

Figure 3. The population of BMC of young (A) and old (B) animals, light microscopy of Carl Zeiss Primo Stari LED (Germany). Fluo-3 fluorescence in BMC of young (C) and old (D) animals, confocal laser scanning microscopy of LSM 510 META Carl Zeiss (Germany), and fluorescence intensity in arbitrary units in BMC obtained from young (E) and old $(\mathrm{F})$ animals, magnification $\times 100$.

indicates the presence of causes leading it to transport into the cells, and on the other hand, suggests that BMC of old animals, rich in calcium, can be altered and other metabolic characteristics of cells. The obtained results and available data [28] allow us to suggest that the BMC microenvironment, which changes with age, affects not only the speed and direction of differentiation of mesenchymal stem cells, but also affects the features of the metabolome and epigenome in finally differentiated cells. As a result, the cells that form in the bone marrow are different from the BMCs that form in young animals, although morphologically these types of cells may be similar. Experimental verification of this assumption is a rather complicated task, since it requires an analysis of the metabolic features of a small number of cells or individual cells.

However, this problem can be solved using the integral approach. If we assume that, the metabolism of lymphocytes or neutrophils in young and old animals is different and on the organism of these animals to have some type of influence that will change the nature of the microenvironment in the bone marrow. Then the response of cells obtained from young and old animals will behave according to differently after transferring them to primary cultures. Since new epigenetic and metabolic characteristics will be formed in the changed BMC 
microenvironment, when such cells are transferred to the primary culture, their behavior will differ from BMC obtained from intact young and old animals.

It was previously shown that induction of liver fibrosis had an age-dependent response to the behavior of BMC in culture [29].

It was found that if the lymphocytes obtained in young animals with $\mathrm{Cu}$-induced liver fibrosis were transferred to culture, they acquired the ability to actively proliferate, unlike the BMC of young animals without liver fibrosis (Figure 4). At the same time, the proliferative activity of lymphocytes obtained from old animals with liver fibrosis remained the same as for old intact animals (Figure 4).

Consequently, the development of liver fibrosis influenced the proliferative activity of lymphocytes in young animals, but not in old animals. These results confirm the assumption about the influence of the microenvironment on the formation of a metabolome on differentiating into BMC and the effect of this influence depends on the initial state of the microenvironment, in the sense that it is initially different in old and young animals.

The definition of the "lifespan" of stab neutrophils in the primary culture obtained in young animals with liver fibrosis did not change as compared with those obtained in young animals without liver fibrosis (Figure 2, Figure 4). It should be noted that the "lifespan" of segmented neutrophils in the primary

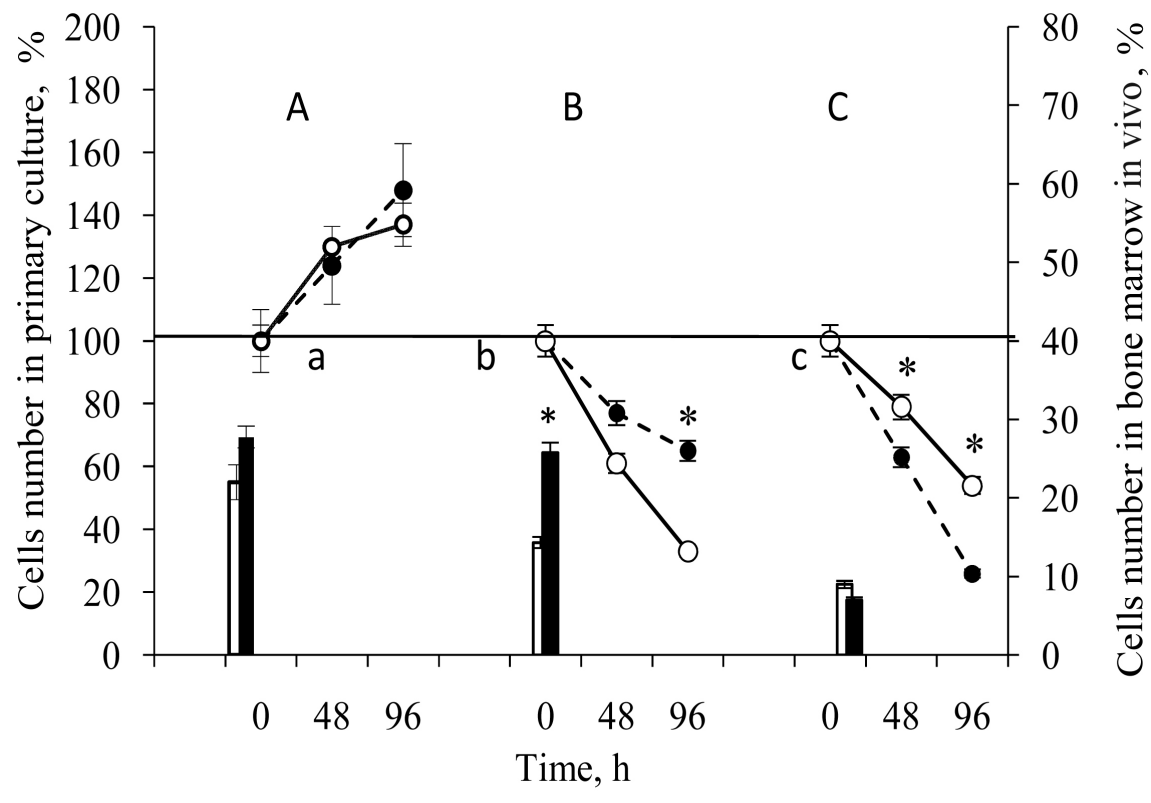

\section{- - - 3 months $\longrightarrow-20$ months}

Figure 4. The number of lymphocytes (A), band neutrophils (B) and segmented neutrophils (C) in the primary culture of BMC at 48 and 96 hours of cultivation obtained from young ( 3 months) and old (20 months) animals with $\mathrm{Cu}$-nduced liver fibrosis, and the number of lymphocytes (a), band neutrophils (b) and segmented neutrophils (c) contained in the bone marrow in vivo of young ( $\square$ ) and old ( $)$ animals $\mathrm{Cu}$-induced liver fibrosis. Data percentage is expressed as mean, obtained of three independent experiments \pm S.E.M, $\mathrm{n}=8$ per group; variants with $\mathrm{p} \leq 0.05$ between young and old animals are determined by ${ }^{*}$, by Mann-Whitney's U-test. 
culture was 30\% compared with the cells of old animals without fibrosis (Figure 2, Figure 4). It should be noted that the "lifespan" of segmented neutrophils in the primary culture decreased if they were obtained in both young and old animals with liver fibrosis, and this was more pronounced in old than in young animals (Figure 4).

Consequently, if BMC (lymphocytes and neutrophils) were formed against the background of liver fibrosis, then their functional characteristics changed, judging by their ability to proliferate and retention time in the primary culture, and this happened differently depending on the age of the animals, i.e. their backstory.

\section{Discussion}

The results of this work can be reduced to several general provisions:

1) the amount of BMC isolated from old (20 months) rats and transferred to the primary culture with the addition of $20 \%$ fetal calf serum increased significantly more compared to the number of BMC isolated from young (3 months) rats. This was particularly pronounced during the first 24 hours of cultivation;

2) such superiority in the proliferative activity of BMC in the primary culture obtained from old animals is associated with the active proliferation of bone marrow lymphocytes and a significant increase in "lifespan" in culture of segmented neutrophils compared with those of young animals;

3 ) in the bone marrow of old animals contained more lymphocytes (by 62.5\%) compared with the bone marrow of young animals;

4) BMC contained 3 times more intracellular calcium compared with the cells of young animals;

5) induction of liver fibrosis in young and old animals, induced by repeated injections of copper sulfate, was accompanied by a pronounced age-dependent response to BMC. Thus, in young animals, fibrosis caused an increase in the number of lymphocytes in the bone marrow by $167 \%$, and in old animals by $26 \%$.

The lymphocytes of young animals formed in the bone marrow against the background of liver fibrosis acquired the ability to proliferate after being transferred to the primary culture and did not differ, by this indicator, from the lymphocytes of old animals. At the same time, the "lifespan" of segmented neutrophils formed on the background of liver fibrosis in old animals was significantly reduced compared with the number of neutrophils obtained in intact old rats.

The results obtained can be explained from the standpoint of the different (age-dependent) effects of the microenvironment on the formation of epigenetic-metabolic patterns in BMC. From this working hypothesis, it follows that morphologically "similar" BMCs may differ in the characteristics of the metabolome and, as a result, behave differently when changing (changing) the microenvironment. In other words, the metabolic heterogeneity of BMC is much more diverse than their morphological variability. 
As known, the bone marrow is a highly vascularized tissue that has a well-developed capillary network [30], so that the BMC microenvironment in the bone marrow changes rapidly and effectively in accordance with the dynamically changing composition of the blood. The intensity of division and the direction of differentiation of BMCs are influenced by the physicochemical characteristics and composition of the cell niche [31]. It can be argued that cytokines play a crucial role in the formation of cell types in the bone marrow. Cytokines are a large group of polypeptide mediators (more than a hundred have been identified) that regulate immunopathy, erythropoiesis and the functioning of the immune system [32].

In fact, the cytokine network determines how many and what types will be formed in the bone marrow. Lymphocytes (B-, T-, NK-cells) provide humoral immunity (antibody synthesis), cellular immunity (cell-cell interactions) and their induction occurs in the presence of pathogens or inflammatory processes in the body.

Thus, the induction of inflammation-liver fibrosis was accompanied by an increase in the number of lymphocytes in the bone marrow of young rats by $167 \%$ compared with the initial number. At the same time, lymphocytes, which were formed on the background of fibrosis, acquired the ability to proliferate in the primary culture.

At the same time, intact old animals the content of lymphocytes in the bone marrow was the same as in young animals with fibrosis. From this, it follows that the seemingly healthy intact old animals probably had some kind of an inflammatory reaction unknown to us. In that case, if liver fibrosis was induced in old animals, the number of lymphocytes in the bone marrow also increased, but only by $26 \%$, compared with the initial number.

It is known that when the liver functions abnormally, the spectrum of cytokines changes, the number of products of free radical reactions increases, and mesenchymal stem cells actively migrate to the focus of inflammation [33]. It can be assumed that such changes lead to changes in the cellular microenvironment in the bone marrow. This is supported by data showing that segmented neutrophils produced on the background of liver fibrosis in old animals had a much shorter lifespan in the primary culture than the same cells formed in old intact animals.

Perhaps the most difficult question in the interpretation of complex, multidirectional responses is different effects in animals of different ages. Therefore, it would seem the same type of induction of liver fibrosis in young and old animals led to different responses in animals of different ages. We believe that the qualitative and quantitative characteristics of the response are determined by the initial state of the system. Thus, the relatively low content of lymphocytes in the bone marrow of young animals was accompanied by their significant increase in the induction of liver fibrosis. At the same time, the initially high content of lymphocytes in old animals was accompanied by their slight increase in the in- 
duction of liver fibrosis. This indicates that the activity of lymphopoiesis has certain limits and a further increase in the number of animal cells is impossible. However, it isn't follows from this that old animals have exhausted possible adaptive responses to external inductors. From this it follows that in this case, in the body of old animals, alternative ways of adaptation can be "used" or, in other words, old animals can use strategies of adaptation that differ from young animal [19]. The data obtained in the present work on neutrophil characteristics may also be in favor of this. Thus, lymphocytes and neutrophils can regulate different parts of the immune response [34], and changes in the ratio of these types of cells indicate the use of different strategies. Differences in the proliferative activity of lymphocytes and the "lifespan" of band neutrophils in the primary culture obtained in young and old animals, based on the proposed working hypothesis, are explained by different metabolic characteristics of the same morphological types of BMC in young and old animals. Therefore, it turned out that the BMC of intact old animals contained 3 times more intracellular calcium as compared with young animals.

It was shown that an increase in the content of intracellular calcium by a factor of 2 - 3 was accompanied by a significant increase in the proliferative activity of the cells. It is well known that calcium is one of the main secondary messengers in the body [35].

Calcium is involved in more than 300 known important biological reactions [36]. An increase in the calcium content in the cell clearly indicates a change in the cells of the entire metabolism, which was manifested in an increase in the proliferative activity of lymphocytes in the primary culture and a change in the "lifespan" of cells in the culture.

Differences in the calcium content in the BMC of old and young animals could be due to the difference in the BMC microenvironment in young and old animals. The mechanisms for this require further research.

\section{Conflicts of Interest}

The authors declare no conflicts of interest regarding the publication of this paper.

\section{References}

[1] Ideker, T., Galitski, T. and Hood, L. (2001) A New Approach to Decoding Life: Systems Biology. Annual Review of Genomics and Human Genetics, 2, 343-372. https://doi.org/10.1146/annurev.genom.2.1.343

[2] Krause, D.S., Fulzele, K., Catic, A., Sun, C.C., Dombkowski, D., Hurley, M.P. Divieti-Pajevic, P., et al. (2013) Differential Regulation of Myeloid Leukemias by the Bone Marrow Microenvironment. Nature medicine, 19, 1513-1517. https://doi.org/10.1038/nm.3364

[3] Johnson, R. and Halder, G. (2014) The Two Faces of Hippo: Targeting the Hippo Pathway for Regenerative Medicine and Cancer Treatment. Nature Reviews Drug Discovery, 13, 63-79. https://doi.org/10.1038/nrd4161 
[4] Atala, A., Lanza, R., Mikos, T. and Nerem, R. (2018) Principles of Regenerative Medicine. Academic Press, Cambridge.

[5] Cooper, S. (2006) Distinguishing between Linear and Exponential Cell Growth during the Division Cycle: Single-Cell Studies, Cell-Culture Studies, and the Object of Cell-Cycle Research. Theoretical Biology and Medical Modelling, 3, 10. https://doi.org/10.1186/1742-4682-3-10

[6] Orlic D., Kajstura J., Chimenti S., et al. (2000) Bone Marrow Cells Regenerate Infarcted Myocardium. Nature, 410, 701-705. https://doi.org/10.1038/35070587

[7] Dong, L., Yu, W.M., Zheng, H., Loh, M.L., Bunting, S.T., Pauly, M., Qu, C K., et al. (2016) Leukaemogenic Effects of Ptpn11 Activating Mutations in the Stem Cell Microenvironment. Nature, 539, 304-308. https://doi.org/10.1038/nature20131

[8] Kumar, V., Patel, S., Tcyganov, E. and Gabrilovich, D.I. (2016) The Nature of Myeloid-Derived Suppressor Cells in the Tumor Microenvironment. Trends in immunology, 37, 208-220. https://doi.org/10.1016/j.it.2016.01.004

[9] Gao, F., Chiu, S.M., Motan, D.A.L., Zhang, Z., Chen, L., Ji, H.L., Lian, Q., et al. (2017) Mesenchymal Stem Cells and Immunomodulation: Current Status and Future Prospects. Cell Death \& Disease, 7, e2062. https://doi.org/10.1038/cddis.2015.327

[10] Rani, S., Ryan, A.E., Griffin, M.D. and Ritter, T. (2015) Mesenchymal Stem cell-Derived Extracellular Vesicles: Toward Cell-Free Therapeutic Applications. Molecular Therapy, 23, 812-823. https://doi.org/10.1038/mt.2015.44

[11] Bozhkov, A.I., Klimova, E.M., Nikitchenko, Y.V., Davydov, V.V., Zvyagintseva, O.V., Kurguzova, N.I., Naglov, A.V., et al. (2014) Stem Cells Take Part in Regulation of Prooxidant Activity and Immunity at Liver Fibrosis. American Journal of Biomedical and Life Sciences, 2, 5-12. https://doi.org/10.11648/j.ajbls.s.2014020601.12

[12] Goodell, M.A. and Rando, T.A. (2015) Stem Cells and Healthy Aging. Science, 350, 1199-1204. https://doi.org/10.1126/science.aab3388

[13] Kusumbe, A.P., Ramasamy, S.K., Itkin, T., Mäe, M.A., Langen, U.H., Betsholtz, C., Adams, R.H., et al. (2016) Age-Dependent Modulation of Vascular Niches for Haematopoietic Stem Cells. Nature, 532, 380-384.

https://doi.org/10.1038/nature17638

[14] Fafián-Labora, J., Fernández-Pernas, P., Fuentes, I., De Toro, J., Oreiro, N., Sangiao-Alvarellos, S., Arufe, M.C., et al. (2015) Influence of Age on Rat Bone-Marrow Mesenchymal Stem Cells Potential. Scientific Reports, 5, Article No. 16765. https://doi.org/10.1038/srep16765

[15] Sardo, V.L., Ferguson, W., Erikson, G.A., Topol, E.J., Baldwin, K.K. and Torkamani, A. (2017) Influence of Donor Age on Induced Pluripotent Stem Cells. Nature Biotechnology, 35, 69-74. https://doi.org/10.1038/nbt.3749

[16] Lv, F.J., Tuan, R.S., Cheung, K.M. and Leung, V.Y. (2014) Concise Review: The Surface Markers and Identity of Human Mesenchymal Stem Cells. Stem Cells, 32, 1408-1419. https://doi.org/10.1002/stem.1681

[17] Guo, Y., Liu, L., Sun, M., Hai, Y., Li, Z. and He, Z. (2015) Expansion and Long-Term Culture of Human Spermatogonial Stem Cells via the Activation of SMAD3 and AKT Pathways. Experimental Biology and Medicine, 240, 1112-1122. https://doi.org/10.1177/1535370215590822

[18] (1986) Council Directive 86/609/EEC of 24 November 1986 on the Approximation of Laws, Regulations and Administrative Provisions of the Member States Regard- 
ing the Protection of Animals Used for Experimental and Other Scientific Purposes. Official Journal L, 358, 0001-0028.

[19] Bozhkov, A.I., Nikitchenko, Y.V., Klimova, E.M., Linkevych, O.S., Lebid, K.M., Al-Bahadli, A.M.M. and Alsardia, M.M.A. (2017) Young and Old Rats Have Different Strategies of Metabolic Adaptation to Cu-Induced Liver Fibrosis. Advances in Gerontology, 7, 41-50. https://doi.org/10.1134/S2079057017010040

[20] Amend, S.R., Valkenburg, K.C. and Pienta, K.J. (2016) Murine Hind Limb Long Bone Dissection and Bone Marrow Isolation. Journal of Visualized Experiments, No. 110. https://doi.org/10.3791/53936

[21] Bozhkov, A.I., Kabachnyy, V.I., Bondar, A.Y., Chumak, I.V. and Kolot, N.V. (2014) Hormesis Effect and the Influence of Ultra-Low Glycosides Doses on the BMC Proliferative Activity in Culture. Journal of Harmonized Research in Pharmacy, 3, 154-166.

[22] Lewandowski, K., Kowalik, M.M., Pawlaczyk, R., Rogowski, J. and Hellmann, A. (2012) Microscopic Examination of Bone Marrow Aspirate in Healthy Adults-Comparison of Two Techniques of Slide Preparation. International Journal of Laboratory Hematology, 34, 254-261. https://doi.org/10.1111/j.1751-553X.2011.01387.x

[23] Merritt, J.E., McCarthy, S.A., Davies, M.P. and Moores, K.E. (1990) Use of Fluo-3 to Measure Cytosolic $\mathrm{Ca}^{2+}$ in Platelets and Neutrophils. Loading Cells with the Dye, Calibration of Traces, Measurements in the Presence of Plasma, and Buffering of Cytosolic $\mathrm{Ca}^{2+}$. Biochemical Journal, 269, 513-519. https://doi.org/10.1042/bj2690513

[24] Chen, C., Tang, Z., Song, Q., Yang, M., Shi, Q. and Weng, Y. (2016) Downregulated microRNA-23b Promotes BMP9-Mediated Osteogenesis in C2C12 Myoblast Cells by Targeting Runx2. Molecular Medicine Reports, 13, 2492-2498. https://doi.org/10.3892/mmr.2016.4814

[25] Quah, B.J., Warren, H.S. and Parish, C.R. (2007) Monitoring Lymphocyte Proliferation in Vitro and in Vivo with the Intracellular Fluorescent Dye Carboxy Fluorescein Diacetate Succinimidyl Ester. Nature Protocols, 2, 2049. https://doi.org/10.1038/nprot.2007.296

[26] Carvalho, L.O., Aquino, E.N., Neves, A.C.D. and Fontes, W. (2015) The Neutrophil Nucleus and Its Role in Neutrophilic Function. Journal of Cellular Biochemistry, 116, 1831-1836. https://doi.org/10.1002/jcb.25124

[27] Görlach, A., Bertram, K., Hudecova, S. and Krizanova, O. (2015) Calcium and ROS: A Mutual Interplay. Redox Biology, 6, 260-271. https://doi.org/10.1016/j.redox.2015.08.010

[28] Bozhkov, A.I., Ivanov, E.G., Kuznetsova, Y.A., Ohiienko, S.L. and Bondar, A.Y. (2017) Copper-Induced Liver Fibrosis Affects the Behavior of BMC in Primary Culture. Frontiers in Biology, 12, 271-279. https://doi.org/10.1007/s11515-017-1458-y

[29] Bozhkov, A.I., Ohiienko, S.L., Kuznetsova, Y.A., Bondar, A.Y., Marchenko, V.P. and Gumennaya, M.S. (2017) Donor Age Affects Behavior and Sensibility of BMC to Copper Ions in Primary Culture. Advances in Gerontology, 7, 336-344. https://doi.org/10.1134/S2079057017040026

[30] Morrison, S.J. and Scadden, D.T. (2014) The Bone Marrow Niche for Haematopoietic Stem Cells. Nature, 505, 327. https://doi.org/10.1038/nature12984

[31] Crane, G.M., Jeffery, E. and Morrison, S.J. (2017) Adult Haematopoietic Stem Cell Niches. Nature Reviews Immunology, 17, 573. https://doi.org/10.1038/nri.2017.53 
[32] Vazquez, M.I., Catalan-Dibene, J. and Zlotnik, A. (2015) B Cells Responses and Cytokine Production Are Regulated by Their Immune Microenvironment. Cytokine, 74, 318-326. https://doi.org/10.1016/j.cyto.2015.02.007

[33] Squillaro, T., Peluso, G. and Galderisi, U. (2016) Clinical Trials with Mesenchymal Stem Cells: An Update. Cell Transplantation, 25, 829-848. https://doi.org/10.3727/096368915X689622

[34] Nathan, C. (2006) Neutrophils and Immunity: Challenges and Opportunities. Nature Reviews Immunology, 6, 173. https://doi.org/10.1038/nri1785

[35] Pornsiriwong, W., Estavillo, G.M., Chan, K.X., Tee, E.E., Ganguly, D., Crisp, P.A., Yong, M.T., et al. (2017) A Chloroplast Retrograde Signal, 3'-Phosphoadenosine 5'-Phosphate, Acts as a Secondary Messenger in Abscisic Acid Signaling in Stomatal Closure and Germination. Elife, 6, e23361. https://doi.org/10.7554/eLife.23361

[36] Prati, C. and Gandolfi, M.G. (2015) Calcium Silicate Bioactive Cements: Biological Perspectives and Clinical Applications. Dental Materials, 31, 351-370.

https://doi.org/10.1016/j.dental.2015.01.004 